\title{
Description of locally finite families from a nonstandard point of view
}

\author{
ULF LEONARD CLOTZ
}

\begin{abstract}
In nonstandard topology we can describe a given topology by the neighbourhood-monads of the standard points (see Sari, chapter 6 in Diener and Diener [2]). Calling an internal point near-standard if it is contained in the neighbourhood-monad of some standard point and remote otherwise we give some statements about locally finite families and paracompact spaces in terms of nearstandard and remote points.
\end{abstract}

2000 Mathematics Subject Classification 03H05 (primary); 54J05 (secondary)

Keywords: nonstandard topology, remote points, locally finite, paracompact

\section{Introduction}

Given some topological space $(X, \mathcal{T})$ in the standard world one can describe such notions as open and closed sets or continuity by the neighbourhood-monads of the standard points in some nonstandard extension. This neighbourhood-monad is defined as the filtermonad of the neighbourhood-filter: Let $x \in X$ and $\mathcal{V}(x):=\{V \in \mathcal{T}: x \in V\}$ be the nbd-filter, then $\mu_{\mathcal{T}}\left({ }^{*} x\right):=\bigcap\left\{{ }^{*} V: V \in \mathcal{V}(x)\right\}$ is called the neighbourhood-monad (or nbd-monad).

The definition of the nbd-monad can be found already in Robinson [6], together with several applications to topological properties. One of the famous applications is the proof that a topological space $(X, \mathcal{T})$ is compact iff every (internal) point of ${ }^{*} X$ is near-standard, i.e. contained in the nbd-monad of some standard point in ${ }^{*} X$. If $X$ is not compact there exist so-called remote points (cf Stroyan - Luxemburg [7]), which don't belong to any nbd-monad of standard points.

We use the notions of near-standard and remote to give a nonstandard description of locally finite families (Lemma 3.2). The main results are Theorem 3.14 and Theorem 3.17 which provide a nonstandard sufficient condition for a regular space to be paracompact. For the proofs of these theorems we use the nonstandard characterization of paracompactness given in [7] (which is here additionally stated using monads 
instead of internal sets, cf Lemma 3.13) and the nonstandard characterization of local finiteness we gave before.

All results are part of the author's PhD-thesis [1] written under the supervision of Professor Dr. Michael Reeken.

\section{Preliminaries}

We are working in the nonstandard set theory HST (see Kanovei and Reeken [4], also for the usage of logical and mathematical symbols). So we have the class WWF in the universe $\mathbb{H}$ which behaves like an ordinary $\mathbf{Z F C}$-universe. For every well-founded $X \in \mathbb{W} \mathbb{F}$ there is a unique standard set $S={ }^{*} X \in \mathbb{S}$. Using Separation we can extract the standard elements from every given set: $\{x \in X$ : st $x\}=X \cap \mathbb{S}=:{ }_{\sigma} X$. A set $X$ is of standard size if there exists a (possibly external) bijection $f$ between $X$ and the standard elements of a standard set $S \in \mathbb{S}$, i.e. $f: X \rightarrow{ }_{\sigma} S$. This is equivalent to the existence of a (possibly external) bijection $f: X \rightarrow W \in \mathbb{W}$ F onto a well-founded set $W$.

For a better orientation in this multiple universe setting we will use roman letters $(X, Y, x, y, \ldots)$ for sets in $\mathbb{W} \mathbb{F}$ and gothic letters $(\mathfrak{A}, \mathfrak{V}, \mathfrak{x}, \ldots)$ for internal sets and external sets with only internal elements.

In HST we can use Saturation in the following form: Every f.i.p. ${ }^{1}$ family $\mathcal{F}$ of standard size which consists only of internal sets has nonempty intersection $\bigcap \mathcal{F} \neq \emptyset$.

Using this, we get one important tool in nonstandard arguing, which is as follows:

Lemma 2.1 (Overspill) Given internal sets $\mathfrak{X}$ and $\mathfrak{Y}$ with ${ }_{\sigma} \mathfrak{X} \subseteq \mathfrak{Y}$, there exists a hyperfinite set $\mathfrak{Z}$ with ${ }_{\sigma} \mathfrak{X} \subseteq \mathfrak{Z} \subseteq \mathfrak{Y}$.

A monad in our sense is the intersection of a standard-sized family of standard sets. Given a filter $\mathcal{F}$ on some set $X \in \mathbb{W} \mathbb{F}$ it follows from Saturation that the intersection $\bigcap\left\{{ }^{*} F: F \in \mathcal{F}\right\}$ is always a nonempty monad. We call this intersection the filtermonad of $\mathcal{F}$.

Given a topological space $(X, \mathcal{T}) \in \mathbb{W} \mathbb{F}$, let $\mathcal{V}$ map any subset $A \subseteq X$ to the set $\mathcal{V}(A)$ of all open sets containing $A$. From this we get a map ${ }^{*} \mathcal{V}$, which maps any internal

\footnotetext{
${ }^{1}$ finite intersection property, i.e. every finite subfamily $\mathcal{F}^{\prime} \subset \mathcal{F}$ of the original family $\mathcal{F}$ has nonempty intersection $\bigcap \mathcal{F}^{\prime} \neq \emptyset$.
} 
subset $\mathfrak{A} \subseteq{ }^{*} X$ to the internal set ${ }^{*} \mathcal{V}(\mathfrak{A})$ of all internal open sets containing $\mathfrak{A}$. For convenience we write $\mathcal{V}(x)$ when $A=\{x\}$ is a singleton.

For any internal subset $\mathfrak{A} \subseteq{ }^{*} X$ the set ${ }_{\sigma}^{*} \mathcal{V}(\mathfrak{A})$ of all standard neighbourhoods of $\mathfrak{A}$ defines a standard filter on ${ }^{*} X$ which is the $*$-image of some filter $\mathcal{F}$ on $X$. We call the filtermonad of $\mathcal{F}$ the neighbourhood-monad of $\mathfrak{A}$ and denote it by $\mu_{\mathcal{T}}(\mathfrak{A})$. Again we write $\mu_{\mathcal{T}}(\mathfrak{x})=\mu_{\mathcal{T}}(\{\mathfrak{x}\})$.

Using the notation given above we get the following well-known nonstandard description of open sets:

$$
A \subseteq X \text { is open iff for every } a \in A \text { we have } \mu_{\mathcal{T}}\left({ }^{*} a\right) \subseteq{ }^{*} A .
$$

In Sari [2, chapter 6] one can find the following equality for internal $\mathfrak{A} \subseteq{ }^{*} X$ :

$$
\mu_{\mathcal{T}}(\mathfrak{A})=\bigcup_{\mathfrak{a} \in \mathfrak{A}} \mu_{\mathcal{T}}(\mathfrak{a})
$$

Using Overspill we get the following:

Lemma 2.2 For any internal subset $\mathfrak{A} \subseteq{ }^{*} X$ there is an internal neighbourhood $\mathfrak{V}$ for which $\mathfrak{A} \subseteq \mathfrak{V} \subseteq \mu_{\mathcal{T}}(\mathfrak{A})$ holds.

If $\overline{\mathfrak{A}}$ denotes the topological closure of $\mathfrak{A} \subseteq{ }^{*} X$ we get by $*$-Transfer

$$
\overline{\mathfrak{A}}=\left\{\mathfrak{x} \in{ }^{*} X: \forall^{\text {int }} \mathfrak{V} \in{ }^{*} \mathcal{V}(\mathfrak{x})(\mathfrak{V} \cap \mathfrak{A} \neq \emptyset)\right\}
$$

for internal $\mathfrak{A}$. For later application we take this equality as a definition for the topological closure of external sets (such as monads). For $A \subseteq X$ we have the condition

$$
\left.x \in \bar{A} \text { iff } \mu_{\mathcal{T}}{ }^{*} x\right) \cap{ }^{*} A \neq \emptyset .
$$

If we take a look at the neighbourhood-monad $\mu_{\mathcal{T}}(\mathfrak{A})$ of an internal subset $\mathfrak{A} \subseteq{ }^{*} X$ we get the following result:

Lemma 2.3 For internal $\mathfrak{A} \subseteq{ }^{*} X$ we have

$$
\overline{\mu_{\mathcal{T}}(\mathfrak{A})}=\bar{\mu}_{\mathcal{T}}(\mathfrak{A}):=\bigcap\left\{\overline{\mathfrak{V}}: \mathfrak{V} \in_{\sigma}^{*} \mathcal{V}(\mathfrak{A})\right\}
$$

Proof For any $\mathfrak{V} \in{ }_{\sigma}^{*} \mathcal{V}(\mathfrak{A})$ we have $\mu_{\mathcal{T}}(\mathfrak{A}) \subseteq \mathfrak{V}$, so $\overline{\mu_{\mathcal{T}}(\mathfrak{A})} \subseteq \overline{\mathfrak{V}}$ and $\overline{\mu_{\mathcal{T}}(\mathfrak{A})} \subseteq \bar{\mu}_{\mathcal{T}}(\mathfrak{A})$ follows.

For $\mathfrak{x} \notin \overline{\mu_{\mathcal{T}}(\mathfrak{A})}$, there exists an internal neighbourhood $\mathfrak{V}$ with $\mathfrak{V} \cap \mu_{\mathcal{T}}(\mathfrak{A})=\emptyset$. But then there exists standard $\mathfrak{W} \in{ }_{\sigma}^{*} \mathcal{V}(\mathfrak{A})$ with $\mathfrak{V} \cap \mathfrak{W}=\emptyset$, so $\mathfrak{x} \notin \overline{\mathfrak{W}} \supseteq \bar{\mu}_{\mathcal{T}}(\mathfrak{A})$. 
Now we can generalize (4):

Lemma 2.4 For $\mathfrak{x} \in{ }^{*} X$ and internal $\mathfrak{A} \subseteq{ }^{*} X$ we have

$$
\mathfrak{x} \in \bar{\mu}_{\mathcal{T}}(\mathfrak{A}) \Longleftrightarrow \mu_{\mathcal{T}}(\mathfrak{x}) \cap \mu_{\mathcal{T}}(\mathfrak{A}) \neq \emptyset .
$$

Proof First, let $\mathfrak{x} \in \bar{\mu}_{\mathcal{T}}(\mathfrak{A})$ and $\mathfrak{V} \subset \mu_{\mathcal{T}}(\mathfrak{x})$ be a neighbourhood of $\mathfrak{x}$, which exists by Lemma 2.2. By (3) and Lemma 2.3 we have $\mathfrak{V} \cap \mu_{\mathcal{T}}(\mathfrak{A}) \neq \emptyset$, so $\mu_{\mathcal{T}}(\mathfrak{x}) \cap \mu_{\mathcal{T}}(\mathfrak{A}) \neq \emptyset$.

Now, let $\mathfrak{x} \notin \bar{\mu}_{\mathcal{T}}(\mathfrak{A})$, so there exists a standard open ${ }^{*} V \in{ }_{\sigma}{ }_{\sigma} \mathcal{V}(\mathfrak{A})$ with $\mathfrak{x} \notin{ }^{*} V$. Then $\mu_{\mathcal{T}}(\mathfrak{A}) \subseteq{ }^{*} V$ and $\mu_{\mathcal{T}}(\mathfrak{x}) \subseteq{ }^{*} X \backslash{ }^{*} \bar{V}$, and it follows $\mu_{\mathcal{T}}(\mathfrak{x}) \cap \mu_{\mathcal{T}}(\mathfrak{A})=\emptyset$.

From (2) we get:

Lemma 2.5 For internal $\mathfrak{A} \subseteq{ }^{*} X$ we have $\bar{\mu}_{\mathcal{T}}(\mathfrak{A})=\bigcup_{\mathfrak{a} \in \mathfrak{A}} \bar{\mu}_{\mathcal{T}}(\mathfrak{a})$.

Proof For $\mathfrak{x} \in \bar{\mu}_{\mathcal{T}}(\mathfrak{A})$ there exists by (2) and Lemma 2.4 some $\mathfrak{a}_{0} \in \mathfrak{A}$ with $\mu_{\mathcal{T}}(\mathfrak{x}) \cap$ $\mu_{\mathcal{T}}\left(\mathfrak{a}_{0}\right) \neq \emptyset$. Applying Lemma 2.4 again gives $\mathfrak{x} \in \bar{\mu}_{\mathcal{T}}\left(\mathfrak{a}_{0}\right) \subseteq \bar{\mu}_{\mathcal{T}}(\mathfrak{A})$.

The remaining subset-relation is obvious.

As we can see in (1) and (4), the neighbourhood-monads of the standard points play a crucial role in nonstandard topology, so we will denote the elements of these monads by a special name:

Definition 2.6 We call $\mathfrak{x} \in{ }^{*} X$ near-standard iff there exists $x \in X$ with $\mathfrak{x} \in \mu_{\mathcal{T}}\left({ }^{*} x\right)$ and remote otherwise. $\mathrm{ns}\left({ }^{*} X\right):=\bigcup_{x \in X} \mu_{\mathcal{T}}\left({ }^{*} x\right)$ denotes the (external) subset of all near-standard points and $\operatorname{rmt}\left({ }^{*} X\right):={ }^{*} X \backslash \mathrm{ns}\left({ }^{*} X\right)$ the subset of all remote points.

Let us illustrate these notions:

Example 2.7 (1) Let $\mathcal{T}$ be the ordinary metric topology on $\mathbb{R}$. Then

$$
\begin{gathered}
\mu_{\mathcal{T}}\left({ }^{*} x\right)=\left\{\mathfrak{x} \in{ }^{*} \mathbb{R}: \mathfrak{x} \approx{ }^{*} x\right\}=\left\{\mathfrak{x}: \forall n \in \mathbb{N}\left(\left|\mathfrak{x}-{ }^{*} x\right|<1 / n\right)\right\} \\
\text { so } \mathrm{ns}\left({ }^{*} \mathbb{R}\right)=\{\mathfrak{x}: \exists n \in \mathbb{N}(|\mathfrak{x}|<n)\} \text { and } \operatorname{rmt}\left({ }^{*} \mathbb{R}\right)=\{\mathfrak{x}: \forall n \in \mathbb{N}(|\mathfrak{x}|>n)\} .
\end{gathered}
$$

(2) More general, if $\Gamma$ is a family of pseudometrics then the monads of the topology generated by $\Gamma$ are

$$
\mu_{\Gamma}\left({ }^{*} x\right)=\left\{\mathfrak{x}: \forall \rho \in \Gamma \forall n \in \mathbb{N}\left(\rho\left(\mathfrak{x},{ }^{*} x\right)<1 / n\right)\right\} .
$$


(3) Now let $\mathcal{S}$ be generated by $\mathcal{T} \cup\{\mathbb{Q}\}$ with $\mathcal{T}$ as above. Then $\mu_{\mathcal{S}}\left({ }^{*} x\right)=\mu_{\mathcal{T}}\left({ }^{*} x\right)$ if $x \in \mathbb{R} \backslash \mathbb{Q}$ and $\mu_{\mathcal{S}}\left({ }^{*} x\right)=\mu_{\mathcal{T}}\left({ }^{*} x\right) \cap{ }^{*} \mathbb{Q}$ if $x \in \mathbb{Q}$. For $\mathfrak{n} \in{ }^{*} \mathbb{N} \backslash \mathbb{N}$ we have $1 / \mathfrak{n} \in \mu_{\mathcal{S}}(0)$, but $\sqrt{2} / \mathfrak{n} \notin \mu_{\mathcal{S}}(0)$ and therefore $\sqrt{2} / \mathfrak{n}$ is a remote point (for $\mathcal{S}$ ).

Now we give three of the separation axioms in terms of nonstandard topology:

Definition 2.8 We call a topological space $(X, \mathcal{T})$

separated iff for all $x \neq y \in X$ we have $\mu_{\mathcal{T}}(* x) \cap \mu_{\mathcal{T}}(* y)=\emptyset$,

regular iff $\mu_{\mathcal{T}}(* x) \cap \mu_{\mathcal{T}}(\mathfrak{y}) \neq \emptyset$ implies $\mathfrak{y} \in \mu_{\mathcal{T}}\left({ }^{*} x\right)$ for arbitrary $x \in X$ and $\mathfrak{y} \in{ }^{*} X$ and

normal iff $\mu_{\mathcal{T}}\left({ }^{*} A\right) \cap \mu_{\mathcal{T}}\left({ }^{*} B\right) \neq \emptyset$ implies $\bar{A} \cap \bar{B} \neq \emptyset$ for all $A, B \subseteq X$.

One connection between separated and regular spaces is as follows:

Lemma 2.9 A separated space $(X, \mathcal{T})$ is regular iff for every remote point $\mathfrak{x} \in \operatorname{rmt}\left({ }^{*} X\right)$ we have $\mu_{\mathcal{T}}(\mathfrak{x}) \subseteq \operatorname{rmt}\left({ }^{*} X\right)$.

Proof First, let $X$ be regular and $\mathfrak{x} \in \operatorname{rmt}\left({ }^{*} X\right)$. Then $\mu_{\mathcal{T}}(\mathfrak{x}) \cap \mu_{\mathcal{T}}\left({ }^{*} x\right)=\emptyset$ for every $x \in X$ because otherwise $\mathfrak{x} \in \mu_{\mathcal{T}}\left({ }^{*} x\right)$ by Definition 2.8 , but this contradicts our assumption.

In order to prove sufficiency of the stated condition let $x \in X, \mathfrak{y} \in{ }^{*} X$ with $\mu_{\mathcal{T}}(\mathfrak{y}) \cap$ $\mu_{\mathcal{T}}\left({ }^{*} x\right) \neq \emptyset$. So $\mathfrak{y}$ cannot be remote and therefore $\mathfrak{y} \in \mu_{\mathcal{T}}\left({ }^{*} y\right)$ for some $y \in X$. Then we have $\mu_{\mathcal{T}}(* y) \cap \mu_{\mathcal{T}}(* x) \neq \emptyset$ and $x=y$ by $X$ being separated.

Example 2.10 Let $\mathcal{S}$ be as in Example 2.7 (3). It is $\sqrt{2} / \mathfrak{n} \in \operatorname{rmt}_{\mathcal{S}}\left({ }^{*} \mathbb{R}\right)$ but for every $V \in \mathcal{S}$ with $\sqrt{2} / \mathfrak{n} \in{ }^{*} V$ it follows from $\sqrt{2} / \mathfrak{n} \in{ }^{*} \mathbb{R} \backslash{ }^{*} \mathbb{Q}$ that $V \in \mathcal{T}$, so $0 \in V$ and finally $0 \in \mu_{\mathcal{S}}(\sqrt{2} / \mathfrak{n}) \not \subset \operatorname{rmt}_{\mathcal{S}}\left({ }^{*} \mathbb{R}\right)$. Thus, $(\mathbb{R}, \mathcal{S})$ is not regular.

If $(X, \mathcal{T})$ is regular, we can conclude from the above lemma together with (2) that for every internal subset $\mathfrak{H} \subseteq \operatorname{rmt}\left({ }^{*} X\right)$ we have $\mu_{\mathcal{T}}(\mathfrak{H}) \subseteq \operatorname{rmt}\left({ }^{*} X\right)$. Applying Lemma 2.4 we even get $\bar{\mu}_{\mathcal{T}}(\mathfrak{H}) \subseteq \operatorname{rmt}\left({ }^{*} X\right)$. This result is not only true for internal subsets of $\operatorname{rmt}\left({ }^{*} X\right)$, but holds also for monads $\mathbf{m} \subseteq \operatorname{rmt}\left({ }^{*} X\right)$, as the next lemma shows.

Lemma 2.11 Let $(X, \mathcal{T})$ be regular and $\mathbf{m} \subseteq \operatorname{rmt}\left({ }^{*} X\right)$ a monad. Then we have $\bar{\mu}_{\mathcal{T}}(\mathbf{m}) \subseteq \operatorname{rmt}\left({ }^{*} X\right)$.

Proof For $X$ being regular, we have $\bar{\mu}_{\mathcal{T}}\left({ }^{*} x\right)=\mu_{\mathcal{T}}\left({ }^{*} x\right)$ for every $x \in X$, so from $\mathbf{m} \subseteq \operatorname{rmt}\left({ }^{*} X\right)$ we know $\bar{\mu}_{\mathcal{T}}\left({ }^{*} x\right) \cap \mathbf{m}=\emptyset$ and there is a neighbourhood $V_{x} \subseteq X$ of $x$ with ${ }^{*} \bar{V}_{x} \cap \mathbf{m}=\emptyset$. So for $U_{x}:=X \backslash \bar{V}_{x}$ it follows $\bar{\mu}_{\mathcal{T}}(\mathbf{m}) \subseteq{ }^{*} \bar{U}_{x}$ and $\mu_{\mathcal{T}}\left({ }^{*} x\right) \cap{ }^{*} \bar{U}_{x}=\emptyset$, from which we conclude $\bar{\mu}_{\mathcal{T}}(\mathbf{m}) \cap \mathrm{ns}\left({ }^{*} X\right)=\emptyset$. 


\section{Local finiteness}

\subsection{A nonstandard description of locally finite families}

Recall the usual definition of local finiteness:

Definition 3.1 A family $\left\{M_{i}: i \in I\right\}$ of subsets of a topological space $(X, \mathcal{T})$ is called locally finite, if for every $x \in X$ there exists some neighbourhood $V_{x}$ such that $V_{x} \cap M_{i} \neq \emptyset$ for only finitely many $i \in I$.

For a locally finite family $\left\{M_{i}: i \in I\right\}$ we look at its $*$-image ${ }^{*}\left\{M_{i}: i \in I\right\}$ which is of the form $\left\{{ }^{*} M(\mathfrak{i}): \mathfrak{i} \in{ }^{*} I\right\}^{2}$ with the standard map ${ }^{*} M:{ }^{*} I \rightarrow{ }^{*} \mathscr{P}(X)$ having the property ${ }^{*} M(* i)={ }^{*}\left(M_{i}\right)$ for every $i \in I$.

Now we can give a nonstandard characterization of local finiteness:

Lemma 3.2 A family $\left\{M_{i}: i \in I\right\} \subseteq \mathscr{P}(X)$ of subsets of a topological space $(X, \mathcal{T})$ is locally finite iff for every near-standard point $\mathfrak{x} \in \mathrm{ns}\left({ }^{*} X\right)$ of ${ }^{*} X$ the set $\left\{\mathfrak{i} \in{ }^{*} I: \mathfrak{x} \in{ }^{*} M(\mathfrak{i})\right\} \subseteq{ }_{\sigma}^{*} I$ contains only standard elements.

Proof First, let $\left\{M_{i}: i \in I\right\}$ be locally finite and $\mathfrak{x} \in \mathrm{ns}\left({ }^{*} X\right)$ some near-standard point, say $\mathfrak{x} \in \mu_{\mathcal{T}}\left({ }^{*} x\right) \subset V_{x}$ with $\left\{i \in I: V_{x} \cap M_{i} \neq \emptyset\right\}$ finite. So we have

$$
\left\{\mathfrak{i} \in{ }^{*} I: \mathfrak{x} \in{ }^{*} M(\mathfrak{i})\right\} \subseteq\left\{\mathfrak{i} \in{ }^{*} I:{ }^{*} V_{x} \cap{ }^{*} M(\mathfrak{i}) \neq \emptyset\right\}={ }^{*}\left\{i \in I: V_{x} \cap M_{i} \neq \emptyset\right\} .
$$

Now this last set is standard and finite and contains therefore only standard elements.

For the reverse implication we assume $\left\{M_{i}: i \in I\right\}$ to be not locally finite, i.e. there is $x_{0} \in X$ such that for every neighbourhood $U_{x_{0}}$ the set $\left\{i \in I: U_{x_{0}} \cap M_{i} \neq \emptyset\right\}$ contains infinitely many elements. In order to show the existence of a near-standard point $\mathfrak{x} \in \mathrm{ns}\left({ }^{*} X\right)$ with $\left\{\mathfrak{i} \in{ }^{*} I: \mathfrak{x} \in{ }^{*} M(\mathfrak{i})\right\} \nsubseteq_{\sigma}^{*} I$ we define the internal sets

$$
\mathfrak{F}_{U, i}:=\left\{(\mathfrak{x}, \mathfrak{i}) \in *^{*}(U \times I \backslash\{i\}): \mathfrak{x} \in{ }^{*} M(\mathfrak{i})\right\}
$$

for $U \in \mathcal{V}\left(x_{0}\right)$ and $i \in I$. Since the f.i.p.-family $\left\{\mathfrak{F}_{U, i}: U \in \mathcal{V}\left(x_{0}\right) \wedge i \in I\right\}$ is of standard size we can apply Saturation and get

$$
\emptyset \neq \bigcap\left\{\mathfrak{F}_{U, i}: U, i\right\}=\left\{(\mathfrak{x}, \mathfrak{i}): \mathfrak{x} \in \mu_{\mathcal{T}}\left({ }^{*} x_{0}\right) \wedge \mathfrak{i} \in{ }^{*} I \backslash{ }_{\sigma}^{*} I \wedge \mathfrak{x} \in{ }^{*} M(\mathfrak{i})\right\} .
$$

Any element in this nonempty intersection now is a near-standard point with the desired property, so the proof is complete.

\footnotetext{
${ }^{2}$ We will use the notation ${ }^{*}\left\{U_{i}: i \in I\right\}=\left\{{ }^{*} U(\mathfrak{i}): \mathfrak{i} \in{ }^{*} I\right\}$ for any family $\left(U_{i}\right)_{i \in I}$ throughout this article.
} 
Example 3.3 Let $(X, d)$ be a metric space and $x_{0} \in X$ some fixed point. The family $\left(U_{n}\right)_{n \in \mathbb{N}}$ for $U_{n}:=\left\{x \in X: d\left(x, x_{0}\right)>n\right\}$ is locally finite:

Let $\mathfrak{x} \in \mathrm{ns}_{d}\left({ }^{*} X\right)$ be arbitrary, say $\mathfrak{x} \in \mu_{d}\left({ }^{*} y\right)=\left\{\mathfrak{y} \in{ }^{*} X:{ }^{*} d\left(\mathfrak{y},{ }^{*} y\right) \approx 0\right\}$ and let $\mathfrak{n} \in$ ${ }^{*} \mathbb{N}$ such that $\mathfrak{x} \in{ }^{*} U(\mathfrak{n})$. Then $\mathfrak{n}<{ }^{*} d\left(\mathfrak{x},{ }^{*} x_{0}\right) \leq{ }^{*} d\left(\mathfrak{x},{ }^{*} y\right)+{ }^{*} d\left({ }^{*} y,{ }^{*} x_{0}\right) \approx{ }^{*}\left(d\left(y, x_{0}\right)\right)$, so $\mathfrak{n} \in \mathbb{N}$, i.e. standard.

Example 3.4 Let $\ell_{1}$ be the space of absolutely summable sequences of real numbers. Then we can identify the space $\ell_{\infty}$ of bounded sequences as its (topological) dual. On $\ell_{\infty}$ we can define $\mathcal{T}$ as the usual topology induced by $\left\|\left(x_{n}\right)_{n}\right\|_{\infty}=\sup \left\{\left|x_{n}\right|: n \in \mathbb{N}\right\}$ and $\mathcal{S}$ as the weak-*-topology. Both topologies are compatible with the linear structure and it suffices to concentrate on the monad of 0 .

$\mu_{\mathcal{T}}(0)=\left\{\mathfrak{h} \in{ }^{*} \ell_{\infty}:\|\mathfrak{h}\|_{\infty} \approx 0\right\}$ and $\mu_{\mathcal{S}}(0)=\left\{\mathfrak{h}: \forall x \in \ell_{1}(\mathfrak{h}(* x) \approx 0)\right\}^{3}$. Now let $\mathfrak{h} \in \mu_{\mathcal{T}}(0)$ and $0 \neq x \in \ell_{1}$ be arbitrary. Taking $\ell_{\infty}$ as the dual of $\ell_{1}$ it follows that $\mathfrak{h}(* x)=\|* x\|_{\infty} \cdot \mathfrak{h}\left({ }^{*} x /\|* x\|_{\infty}\right) \approx 0$, so $\mathfrak{h} \in \mu_{\mathcal{S}}(0)$. Now let $\mathfrak{N} \in{ }^{*} \mathbb{N} \backslash \mathbb{N}$ and $\mathfrak{g}:=\left(\delta_{\mathfrak{N k}}\right)_{\mathfrak{k} \in{ }^{*} \mathbb{N}} \in{ }^{*} \ell_{\infty}$ i.e. $\mathfrak{g}_{\mathfrak{k}}=0$ for $\mathfrak{k} \neq \mathfrak{N}$ and $\mathfrak{g}_{\mathfrak{N}}=1$. For $x=\left(x_{n}\right)_{n} \in \ell_{1}$ we get $\mathfrak{g}(* x)=* x(\mathfrak{N}) \approx 0$ (because $x_{n} \rightarrow 0$ for $n \rightarrow \infty$ ), so $\mathfrak{g} \in \mu_{\mathcal{S}}(0)$. But $\mathfrak{g} \notin \mu_{\mathcal{T}}(0)$, because $\|\mathfrak{g}\|_{\infty}=1$ and for $\mathcal{S}$ is separated and coarser than $\mathcal{T}$ we get that $\mathfrak{g} \in \operatorname{rmt}_{\mathcal{T}}\left({ }^{*} \ell_{\infty}\right)$. In particular, it follows that $\mu_{\mathcal{T}}(0) \varsubsetneqq \mu_{\mathcal{S}}(0)$.

Now let $M_{n}:=\left\{f \in \ell_{\infty}: f_{n}=1 \wedge \forall k<n\left(f_{k}=0\right)\right\}$. The family $\left(M_{n}\right)_{n \in \mathbb{N}}$ is locally finite for $\mathcal{T}$ but not for $\mathcal{S}$ : Let $\mathfrak{h} \in \mathrm{ns}_{\mathcal{T}}\left({ }^{*} \ell_{\infty}\right)$, say $\mathfrak{h} \in \mu_{\mathcal{T}}\left({ }^{*} f\right)$ and let $\mathfrak{h} \in{ }^{*} M(\mathfrak{n})$ for $\mathfrak{n} \in{ }^{*} \mathbb{N}$. Assume that $\mathfrak{n} \notin \mathbb{N}$, then ${ }^{*} f(\mathfrak{k}) \approx 0$ for all $\mathfrak{k} \in \mathbb{N}$, so $f=0$ in contrary to ${ }^{*} f(\mathfrak{n}) \approx \mathfrak{h}_{\mathfrak{n}}=1$. So $\mathfrak{n}$ has to be standard and $\left(M_{n}\right)_{n}$ is locally finite for $\mathcal{T}$. Now, let $\mathfrak{g}$ be as above, then $\mathfrak{g} \in \mathrm{ns}_{\mathcal{S}}\left({ }^{*} \ell_{\infty}\right)$ and $\mathfrak{g} \in{ }^{*} M(\mathfrak{N})$ for $\mathfrak{N} \in{ }^{*} \mathbb{N} \backslash \mathbb{N}$, so $\left(M_{n}\right)_{n}$ is not locally finite for $\mathcal{S}$.

Inspection of the proof of Lemma 3.2 indeed gives the next result.

Corollary 3.5 A family $\left\{M_{i}: i \in I\right\} \subseteq \mathscr{P}(X)$ of subsets of a topological space $(X, \mathcal{T})$ is locally finite iff for every $x \in X$ there is a neighbourhood $V_{x} \subseteq X$ with ${ }^{*} V_{x} \cap\left(\bigcup_{\mathfrak{i} \in{ }^{*} I I_{\sigma}^{*} I}{ }^{*} M(\mathfrak{i})\right)=\emptyset$.

\subsection{Application of the nonstandard characterization}

We apply the above characterization of local finiteness on two well-known facts.

\footnotetext{
${ }^{3}$ cf Example 10.1.31 in Stroyan - Luxemburg [7]
} 
Lemma 3.6 If $\left\{M_{i}: i \in I\right\}$ is locally finite, then so is the family $\left\{\bar{M}_{i}: i \in I\right\}$.

Proof Let $\mathfrak{x} \in \mathrm{ns}\left({ }^{*} X\right)$ be an arbitrary near-standard point, say $\mathfrak{x} \in \mu_{\mathcal{T}}\left({ }^{*} x_{0}\right)$ for $x_{0} \in X$, and $\mathfrak{i} \in{ }^{*} I$ such that $\mathfrak{x} \in{ }^{*} \bar{M}(\mathfrak{i})$. By Overspill there is an internal neighbourhood $\mathfrak{V}$ of $\mathfrak{x}$ with $\mathfrak{V} \subseteq \mu_{\mathcal{T}}\left({ }^{*} x_{0}\right)$. From $\mathfrak{V} \cap{ }^{*} M(\mathfrak{i}) \neq \emptyset$ (see formula (3)) it follows that $\mu_{\mathcal{T}}\left({ }^{*} x_{0}\right) \cap{ }^{*} M(\mathfrak{i}) \neq \emptyset$ which leads to $\mathfrak{i} \in{ }_{\sigma}^{*} I$ by Lemma 3.2.

Lemma 3.7 For a locally finite family $\left\{A_{i}: i \in I\right\}$ of closed subsets $A_{i} \subseteq X$ of a topological space $(X, \mathcal{T})$ the union $\bigcup\left\{A_{i}: i \in J\right\}$ is closed in $X$, where $J \subseteq I$ is any subset of $I$.

Proof Let $\left\{A_{i}: i \in I\right\}$ and $J$ be as in the lemma and $x_{0} \in X$ an arbitrary point with $\mu_{\mathcal{T}}\left({ }^{*} x_{0}\right) \cap *\left(\bigcup\left\{A_{i}: i \in J\right\}\right) \neq \emptyset$. Because of the equality

$$
{ }^{*}\left(\bigcup\left\{A_{i}: i \in J\right\}\right)=\bigcup\left\{{ }^{*} A(\mathfrak{i}): \mathfrak{i} \in{ }^{*} J\right\}
$$

there is some $\mathfrak{j} \in{ }^{*} J$ with $\mu_{\mathcal{T}}\left({ }^{*} x_{0}\right) \cap{ }^{*} A(\mathfrak{j}) \neq \emptyset$. Since the family $\left\{A_{i}: i \in I\right\}$ is locally finite it follows that $\mathfrak{j} \in{ }_{\sigma}^{*} J$ has to be standard, say $\mathfrak{j}=*^{*} j_{0}$ for some $j_{0} \in J$, and so we get $x \in A\left(j_{0}\right) \subseteq \bigcup\left\{A_{i}: i \in J\right\}$ from (4).

To apply these results about locally finite families, we now turn to paracompact spaces. First, we recall the traditional definition in standard terms, which can be found as first definition in Chapter 6.4 of Munkres [5]. After this, we give the nonstandard formulation of paracompactness, which can be found in Stroyan-Luxemburg [7]. After two short proofs of well-known (standard) properties of paracompact spaces we turn to a reformulation of the definition which talks about monads instead of internal sets. This is done in order to give an easy proof of Lemma 3.15 and Lemma 3.16, respectively.

Definition 3.8 (cf [5, Definition in Ch. 6.4]) A separated space $(X, \mathcal{T})$ is paracompact, if each open covering of $X$ has an locally finite open refinement, that covers $X$.

Theorem 3.9 (cf [7, Theorem 8.3.16]) A separated space $(X, \mathcal{T})$ is paracompact iff for every internal subset $\mathfrak{K} \subseteq \operatorname{rmt}\left({ }^{*} X\right)$ there exists an open locally finite covering $\left(U_{i}\right)_{i \in I}$ of $X$ with $\mathfrak{K} \cap\left(\bigcup_{i \in I}^{*}\left(U_{i}\right)\right)=\emptyset$.

An easy consequence of Theorem 3.9 and Lemma 2.9 is the following:

Lemma 3.10 Every paracompact space is regular. 
Example 3.11 (1) $(\mathbb{R}, \mathcal{T})$ with $\mathcal{T}$ as in Example 2.7 (1) is paracompact: For any monad $\mathbf{m} \subset \operatorname{rmt}_{\mathcal{T}}\left({ }^{*} \mathbb{R}\right)$ we can take the following open locally finite covering $\left(U_{n}\right)_{n \in \mathbb{N}_{0}}: U_{0}:=\{x \in \mathbb{R}:|x|<1\}$ and $U_{n}:=\{x \in \mathbb{R}: n-1<|x|<n+1\}$ for $n \geq 1$. From Example 2.7 (1) it follows that $*\left(U_{n}\right) \cap \mathbf{m}=\emptyset$ and that $\mathfrak{n} \in \mathbb{N}$ if $\mathfrak{x} \in{ }^{*} U(\mathfrak{n})$ for $\mathfrak{x} \in \operatorname{rmt}_{\mathcal{T}}\left({ }^{*} \mathbb{R}\right)$.

(2) Let $\mathcal{S}$ be as in Example 2.7 (3), then $(\mathbb{R}, \mathcal{S})$ is not paracompact, since it is not regular (cf Example 2.10).

Another well-known fact about paracompact spaces is as follows:

Lemma 3.12 Let $(X, \mathcal{T})$ be paracompact and $A \subseteq X$ closed. Then $\left(A,\left.\mathcal{T}\right|_{A}\right)$ is paracompact as well.

Proof Let $\mathfrak{H} \subseteq \operatorname{rmt}\left({ }^{*} A\right)$ be any internal subset. Since $\operatorname{rmt}\left({ }^{*} A\right)=\operatorname{rmt}\left({ }^{*} X\right) \cap{ }^{*} A$ there exists an open locally finite covering $\left(U_{i}\right)_{i \in I}$ of $X$ with ${ }^{*}\left(U_{i}\right) \cap \mathfrak{H}=\emptyset$ for all $i \in I$. Let $V_{i}:=U_{i} \cap A$, then $\left(V_{i}\right)_{i \in I}$ is a $\left.\mathcal{T}\right|_{A}$-open covering of $A$ with ${ }^{*}\left(V_{i}\right) \cap \mathfrak{H}=\emptyset$ for all $i \in I$. For $\mathfrak{i} \in{ }^{*} I \backslash{ }_{\sigma}^{*} I$ we have ${ }^{*} V(\mathfrak{i})={ }^{*} U(\mathfrak{i}) \cap{ }^{*} A \subseteq \operatorname{rmt}\left({ }^{*} X\right) \cap{ }^{*} A=\operatorname{rmt}\left({ }^{*} A\right)$ by Lemma 3.2, so $\left(V_{i}\right)_{i \in I}$ is locally finite again by Lemma 3.2.

A characterization of paracompactness in terms of monads instead of internal sets is given by the next lemma.

Lemma 3.13 A separated space $(X, \mathcal{T})$ is paracompact if and only if for every monad $\mathbf{m} \subseteq \operatorname{rmt}\left({ }^{*} X\right)$ there exists an open, locally finite covering $\left(U_{i}\right)_{i \in I}$ of $X$ with ${ }^{*}\left(U_{i}\right) \cap \mathbf{m}=$ $\emptyset$ for all $i \in I$.

The above statement is an immediate consequence of the following stronger result.

Theorem 3.14 Let $(X, \mathcal{T})$ be a topological space and $P$ one of the properties being arbitrary, being open, being closed. Then the following are equivalent:

(1) For every internal subset $\mathfrak{H} \subseteq \operatorname{rmt}\left({ }^{*} X\right)$ there exists a locally finite covering $\left(A_{i}\right)_{i \in I}$ of $X$ with $\mathfrak{H} \cap{ }^{*}\left(A_{i}\right)=\emptyset$ for all $i \in I$ and $A_{i} \subseteq X$ being subsets with property $P$.

(2) For every monad $\mathbf{m} \subseteq \operatorname{rmt}\left({ }^{*} X\right)$ there exists a locally finite covering $\left(A_{i}\right)_{i \in I}$ of $X$ with $\mathbf{m} \cap{ }^{*}\left(A_{i}\right)=\emptyset$ for all $i \in I$ and $A_{i} \subseteq X$ being subsets with property $P$. 
Proof First we show $(i i) \Rightarrow(i)$. Given $\mathfrak{H} \subseteq \operatorname{rmt}\left({ }^{*} X\right)$ internal we have $\delta(\mathfrak{H}) \subseteq$ $\operatorname{rmt}\left({ }^{*} X\right)$ for the discrete monad $\delta(\mathfrak{H}):=\bigcap\left\{{ }^{*} M: M \subseteq X \wedge \mathfrak{H} \subseteq{ }^{*} M\right\}$ (eg, see chapter 8 in Stroyan-Luxemburg [7]).

For $(i) \Rightarrow$ (ii) let $\mathbf{m} \subseteq \operatorname{rmt}\left({ }^{*} X\right)$ be an arbitrary monad. Then for every $x \in X$ there exists an open neighbourhood $V_{x}$ with ${ }^{*}\left(V_{x}\right) \cap \mathbf{m}=\emptyset$. By Standardisation we get ${ }^{*} V(\mathfrak{x})$ for every $\mathfrak{x} \in X$ and by Overspill there is a hyperfinite subset $\mathfrak{X} \subseteq{ }^{*} X$ with ${ }_{\sigma}^{*} X \subseteq \mathfrak{X}$. Now $\mathfrak{W}:={ }^{*} X \backslash \bigcup\left\{{ }^{*} V(\mathfrak{x}): \mathfrak{x} \in \mathfrak{X}\right\} \subseteq \operatorname{rmt}\left({ }^{*} X\right)$ is internal and we find a locally finite covering $\left(A_{i}\right)_{i \in I}$ with $A_{i}$ having property $\mathrm{P}$ and ${ }^{*}\left(A_{i}\right) \cap \mathfrak{W}=\emptyset$ for all $i \in I$. By $*$-Transfer we get a finite subset $Y \subseteq X$ with $A_{i} \subseteq \bigcup_{x \in Y} V_{x}$ for all $i \in I$. It follows ${ }^{*}\left(A_{i}\right) \subseteq{ }^{*}\left(\bigcup_{x \in Y} V_{x}\right)=\bigcup_{x \in Y}{ }^{*}\left(V_{x}\right)$ and finally ${ }^{*}\left(A_{i}\right) \cap \mathbf{m}=\emptyset$ for all $i \in I$.

This equivalence motivates the following two lemmata.

Lemma 3.15 Let $(X, \mathcal{T})$ be a regular space such that for every monad $\mathbf{m} \subseteq \operatorname{rmt}\left({ }^{*} X\right)$ a locally finite covering $\left(U_{i}\right)_{i \in I}$ of $X$ exists with ${ }^{*}\left(U_{i}\right) \cap \mathbf{m}=\emptyset$ for all $i \in I$. Then for every monad $\mathbf{m}^{\prime} \subseteq \operatorname{rmt}\left({ }^{*} X\right)$ there exists a closed locally finite covering $\left(A_{i}\right)_{i \in I}$ of $X$ with ${ }^{*}\left(A_{i}\right) \cap \mathbf{m}^{\prime}=\emptyset$ for all $i \in I$.

Proof Let the monad $\mathbf{m} \subseteq \operatorname{rmt}\left({ }^{*} X\right)$ be arbitrary. By Lemma 2.11 we have $\mu_{\mathcal{T}}(\mathbf{m}) \subseteq$ $\operatorname{rmt}\left({ }^{*} X\right)$ and there is a locally finite cover $\left(U_{i}\right)_{i \in I}$ of $X$ with ${ }^{*}\left(U_{i}\right) \cap \mu_{\mathcal{T}}(\mathbf{m})=\emptyset$ for all $i \in I$. It follows that ${ }^{*}\left(\bar{U}_{i}\right) \cap \mathbf{m}=\emptyset$ and from Lemma 3.6 we know that $\left(\bar{U}_{i}\right)_{i \in I}$ is locally finite.

Lemma 3.16 Let $(X, \mathcal{T})$ be a regular space such that for every monad $\mathbf{m} \subseteq \operatorname{rmt}\left({ }^{*} X\right)$ a closed locally finite covering $\left(U_{i}\right)_{i \in I}$ of $X$ exists with ${ }^{*}\left(U_{i}\right) \cap \mathbf{m}=\emptyset$ for all $i \in I$. Then for every monad $\mathbf{m}^{\prime} \subseteq \operatorname{rmt}\left({ }^{*} X\right)$ there exists an open locally finite covering $\left(O_{i}\right)_{i \in I}$ of $X$ with $^{*}\left(O_{i}\right) \cap \mathbf{m}^{\prime}=\emptyset$ for all $i \in I$.

Proof Again, let the monad $\mathbf{m} \subseteq \operatorname{rmt}\left({ }^{*} X\right)$ be arbitrary. By Lemma 2.11 we have $\bar{\mu}_{\mathcal{T}}(\mathbf{m}) \subseteq \operatorname{rmt}\left({ }^{*} X\right)$ and there exists a locally finite cover $\left(A_{i}\right)_{i \in I}$ of $X$ with ${ }^{*}\left(A_{i}\right) \cap$ $\bar{\mu}_{\mathcal{T}}(\mathbf{m})=\emptyset$ for all $i \in I$. Then for every $i \in I$ there is an open $V_{i}^{\prime} \subseteq X$ with $\mathbf{m} \subseteq{ }^{*} V_{i}^{\prime}$ and $A_{i} \cap \bar{V}_{i}^{\prime}=\emptyset$, so for $V_{i}:=X \backslash \bar{V}_{i}^{\prime}$ we have $A_{i} \subseteq V_{i}$ and ${ }^{*} V_{i} \cap \mathbf{m}=\emptyset$.

Because $\left(A_{i}\right)_{i}$ is locally finite, by Corollary 3.5 there exists open $V_{x} \ni x$ with ${ }^{*} V_{x} \cap$ $\left(\bigcup_{\mathfrak{i} \in{ }^{*} I{ }_{\sigma}{ }^{*} I}{ }^{*} A(i)\right)=\emptyset$ for every $x \in X$. So, $\mathbf{m}_{1}:=\bigcap_{x \in X}{ }^{*}\left(X \backslash V_{x}\right) \subseteq \operatorname{rmt}\left({ }^{*} X\right)$ is a monad with $\bigcup_{\mathfrak{i} \in{ }^{*} I_{\sigma}{ }^{*} I}{ }^{*} A(\mathfrak{i}) \subseteq \mathbf{m}_{1}$. Again, there is a closed locally finite cover $\left(B_{k}\right)_{k \in J}$ of $X$ with ${ }^{*} B_{k} \cap \mathbf{m}_{1}=\emptyset$ for all $k \in J$, so ${ }^{*} B_{k} \cap\left(\bigcup_{\mathfrak{i} \in{ }^{*} I_{\sigma}{ }^{*} I}^{*} A(\mathfrak{i})\right)=\emptyset$. 
Now we set $O_{i}:=X \backslash \bigcup\left\{B_{k}: B_{k} \cap A_{i}=\emptyset\right\}$ which is open by Lemma 3.7. We prove local finiteness of $\left(O_{i}\right)_{i}$ using Lemma 3.2. So let $\mathfrak{x} \in \mathrm{ns}\left({ }^{*} X\right)$ be arbitrary with $\mathfrak{x} \in{ }^{*} O(\mathfrak{i})$. Then $\mathfrak{x} \in{ }^{*} B(\mathfrak{k})$ for $\mathfrak{k} \in{ }^{*} J$ implies ${ }^{*} B(\mathfrak{k}) \cap{ }^{*} A(\mathfrak{i}) \neq \emptyset$. So by Lemma 3.2 we have $\mathfrak{k} \in{ }_{\sigma}^{*} J$ standard and therefore $\mathfrak{i} \in{ }^{*} I$ standard. Finally, if we set $U_{i}:=O_{i} \cap V_{i}$, we get an open locally finite cover $\left(U_{i}\right)_{i \in I}$ of $X$ with ${ }^{*} U_{i} \cap \mathbf{m}=\emptyset$ for all $i \in I$.

Combining Theorem 3.14, Lemma 3.15 and Lemma 3.16, we get the following result. A standard version of this can be found as Theorem 2.3 in chapter VIII in Dugundji [3].

Theorem 3.17 Let $(X, \mathcal{T})$ be a regular space such that for every internal $\mathfrak{H} \subseteq \operatorname{rmt}\left({ }^{*} X\right)$ there is a locally finite covering $\left(U_{i}\right)_{i \in I}$ of $X$ (the $U_{i}$ being arbitrary) with ${ }^{*}\left(U_{i}\right) \cap \mathfrak{H}=\emptyset$ for all $i \in I$. Then $X$ is paracompact.

We use this result to give a nonstandard proof of another well-known fact about paracompact spaces (eg, see [3, chapter VIII, Theorem 2.5]).

Theorem 3.18 Let $A=\bigcup_{k \in \mathbb{N}} A_{k}$ be a countable union of closed subsets $A_{k} \subseteq X$ of the paracompact space $(X, \mathcal{T})$. Then $\left(A,\left.\mathcal{T}\right|_{A}\right)$ is paracompact as well.

Proof Let $\mathfrak{H} \subseteq \operatorname{rmt}\left({ }^{*} A\right)$ be internal. For every $n \in \mathbb{N}$ we have $\mathfrak{H}_{n}:=\mathfrak{H} \cap{ }^{*} A_{n}$ internal with $\mathfrak{H}_{n} \subseteq \operatorname{rmt}\left({ }^{*} A_{n}\right)=\operatorname{rmt}\left({ }^{*} X\right) \cap{ }^{*} A_{n}$, so there is an open locally finite covering $(V(n, i))_{i \in I_{n}}$ of $X$ with ${ }^{*}(V(n, i)) \cap \mathfrak{H}_{n}=\emptyset$ for all $i \in I_{n}$. Let $W_{n}^{\prime}:=\bigcup_{i \in I_{n}} V(n, i)$, then $W_{n}:=W_{n}^{\prime} \backslash \bigcup_{k<n} W_{k}^{\prime}$ and finally $U(n, i):=V(n, i) \cap W_{n}^{\prime} \cap A_{n}$.

For $\mathfrak{a} \in \mathrm{ns}\left({ }^{*} A\right)$, say $\mathfrak{a} \in \mu_{\left.\mathcal{T}\right|_{A}}\left({ }^{*} a\right)$, let $n:=\min \left\{k \in \mathbb{N}: a \in A_{k}\right\}$, then we have $\mathfrak{a} \in{ }^{*}\left(W_{n}^{\prime}\right)$, so $\mathfrak{a} \notin{ }^{*} W(\mathfrak{m})$ for all $\mathfrak{m}>{ }^{*} n$. Therefore $\mathfrak{n} \in{ }^{*} \mathbb{N}$ must be standard if $\mathfrak{a} \in{ }^{*} U(\mathfrak{n}, \mathfrak{i})$ and then $\mathfrak{i} \in{ }^{*} I$ must be standard by $\mathfrak{a} \in{ }^{*} V(\mathfrak{n}, \mathfrak{i})$.

From this it follows that $(U(n, i))_{(n, i)}$ is a locally finite covering of $A$ with ${ }^{*}(U(n, i)) \cap$ $\mathfrak{H}=\emptyset$ for all $n \in \mathbb{N}$ and all $i \in I_{n}$. An application of Theorem 3.17 now completes the proof.

\section{References}

[1] UL Clotz, Untersuchungen einiger topologischer Sachverhalte und Konstruktionen in HST, PhD thesis, Bergische Universität Wuppertal (2009) Available at http://nbn-resolving. de/urn/resolver.pl?urn=urn86 
[2] F Diener, M Diener (editors), Nonstandard Analysis in Practice, Springer-Verlag, Berlin (1995)

[3] J Dugundji, Topology, Allyn and Bacon Inc., Boston, Mass. (1966)

[4] V Kanovei, M Reeken, Nonstandard Analysis, Axiomatically, Springer Monographs in Mathematics, Springer-Verlag, Berlin (2004)

[5] J R Munkres, Topology: A First Course, Prentice-Hall Inc., Englewood Cliffs, N.J. (1975)

[6] A Robinson, Non-standard Analysis, revised edition, North-Holland Publishing Company, Amsterdam (1974)

[7] K D Stroyan, W A J Luxemburg, Introduction to the Theory of Infinitesimals, Academic Press [Harcourt Brace Jovanovich Publishers], New York (1976), Pure and Applied Mathematics, No. 72

Department of Mathematics, University of Wuppertal

Gaußstraße 20, 42119 Wuppertal, Germany

clotz@math.uni-wuppertal.de

http://www . math. uni-wuppertal. de/ clotz

Received: 30 June 2009 Revised: 22 March 2010 